\title{
Estudio comparativo de variables clínicas y costo de la técnica quirúrgica de histerectomía por vía abdominal y laparoscópica
}

\author{
HUGO SALINAS(1), BENJAMÍN NARANJO(1), JORGE PASTÉN(1), BEATRIZ RETAMALES(2), \\ GONZALO DÍAZ(2) y LENKA FRANULIC(2).
}

\section{RESUMEN}

El objetivo de este estudio es comparar los efectos clínicos y económicos de la histerectomía abdominal versus la histerectomía laparoscópica. Es un estudio retrospectivo observacional de 1.663 pacientes de 27 a 83 años histerectomizadas por patología ginecológica benigna durante 1997-2005, en el Hospital Clínico de la Universidad de Chile.

De las 1.663 cirugías, el 11,36\% fueron histerectomías laparoscópicas y el 88,63\% correspondió a histerectomías abdominales. La tasa global de complicaciones fue de 42,8 por 100 mujeres para la histerectomía abdominal y 16,4 para la histerectomía laparoscópica.

La histerectomía laparoscópica presenta algunas ventajas al compararla con la histerectomía abdominal. Se asoció a menos complicaciones y menor tiempo de estadía hospitalaria. En contraste, su costo es significativamente mayor.

Palabras clave: métodos de histerectomía, histerectomía laparoscópica, histerectomía abdominal, costo-efectividad histerectomías.

\section{ABSTRACT}

A COMPARATIVE STUDY OF CLINICAL VARIABLES AND SURGICAL TECHNIQUE COST BETWEEN PATIENTS TREATED WITH EITHER ABDOMINAL OR LAPAROSCOPIC HYSTERECTOMY

The purpose of the present study is to compare clinical and economic outcomes of abdominal versus laparoscopic hysterectomy. The study design was retrospective and observational, and it was conducted on 1.663 patients aged between 27 and 83 undergoing hysterectomy for benign gynecologic conditions between 1997-2005, at the Hospital Clinico of the University of Chile. Of the 1.663 surgeries, 11,36\% were laparoscopic hysterectomies and 88,63\% accounted for abdominal hysterectomies. The global complication rate was 42,8\% for abdominal hysterectomy and 16,4\% for laparoscopic hysterectomy.

Laparoscopic hysterectomy offers advantages such as a fewer complications and a shorter hospital stay when compared to abdominal hysterectomy. However, its cost is significantly higher than that of the abdominal approach.

Keywords: hysterectomy approaches, laparoscopic hysterectomy, abdominal hysterectomy, hysterectomy cost-benefit.

(1) Departamento de Obstetricia y Ginecología. Hospital Clínico de la Universidad de Chile. hsalinas@redclinicauchile.cl (2) Interno(a) de Medicina. Hospital Clínico de la Universidad de Chile. 


\section{INTRODUCCIÓN}

La histerectomía es una intervención quirúrgica frecuente y a la vez costosa en salud. Es una de las cirugías más comúnmente realizadas $^{1-3}$. Después de la operación cesárea, es el segundo procedimiento quirúrgico más frecuente en ginecología.

La oportunidad de una mujer de tener una histerectomía depende de una multiplicidad de factores, incluyendo su edad, la raza, el lugar de residencia y la experiencia de su médico. En prácticamente todos los estudios, el mioma uterino es la principal indicación para la histerectomía ${ }^{4}$. En los últimos años, ha ido ganando terreno la técnica quirúrgica laparoscópica.

Cuando es posible realizar, la histerectomía vaginal (HV) es la ruta de elección, ya que es la alternativa quirúrgica más rápida y más barata, sin otras diferencias claras en los resultados a mediano y largo plazo ${ }^{5}$.

Como es de suponer, las indicaciones para cada vía de abordaje se superponen y no existen criterios específicos que se puedan utilizar para determinar la vía de la histerectomía. La vía de acceso elegida debe ser individualizada. Por todo lo anterior, se hace necesario medir los efectos médicos y económicos de las distintas alternativas quirúrgicas existentes para la resección uterina ${ }^{6}$.

Es razonable pensar que cada una de estas técnicas presenta ventajas y desventajas, dependiendo del motivo por el cual está indicada. Debido a la gran diversidad de criterios existentes por parte de los ginecólogos para optar por cada una de ellas, resulta difícil establecer una comparación directa entre las técnicas quirúrgicas ${ }^{7}$.

Se ha descrito que la histerectomía laparoscópica (HL) tiene numerosas ventajas respecto de la histerectomía abdominal (HA), específicamente, una estadía hospitalaria más corta, un retorno precoz a las actividades normales y una menor morbilidad asociada ${ }^{8}$.

Por el contrario, existen estudios que no encuentran diferencias significativas en el postoperatorio como tampoco una mejor calidad de vida posterior a la cirugía 9 .
No obstante, la HL es ampliamente aceptada como alternativa a la vía abdominal cuando la vía vaginal se considera dificultosa o inapropiada ${ }^{10-21}$.

La diferencia de costos de la histerectomía abdominal en relación a la histerectomía laparoscópica, ha sido establecida sólo en pequeños estudios observacionales y en trabajos con series poco importantes $22-27$.

Los resultados de estos trabajos parecen inconsistentes, puesto que en algunos se encuentra que es más costosa la HL y en otros la $\mathrm{HA}^{27}$.

En el Hospital Clínico de la Universidad de Chile, la HL se efectúa de la forma tradicional, realizando la resección uterina en su totalidad por vía abdominal, no existiendo experiencia con la modalidad laparoscópica con asistencia vaginal referida en la literatura anglosajona.

El objetivo de nuestro trabajo es evaluar y comparar la HA con la HL realizadas en nuestro Departamento de Obstetricia y Ginecología, considerando variables epidemiológicas, médicas, quirúrgicas y postquirúrgicas, además de contrastar el costo de producción de cada intervención con los valores reales de las histerectomías realizadas en el período estudiado.

\section{MATERIAL Y MÉTODO}

Se realizó un estudio retrospectivo observacional de las pacientes sometidas a histerectomía en el Departamento de Obstetricia y Ginecología del Hospital Clínico de la Universidad de Chile. Se compara las dos modalidades de histerectomía (HA vs HL) indicadas por patología ginecológica benigna durante el período comprendido entre enero del año 1997 hasta diciembre del 2005. Cada paciente fue ingresada a una base de datos File Maker 8.0 previo a la cirugía, creando campos con distintas variables epidemiológicas, cuantitativas y cualitativas.

Se analiza las distintas variables relacionadas al procedimiento quirúrgico en si, es decir, tiempo operatorio y complicaciones durante el procedimiento; específicamente pérdidas sanguíneas, lesión vesical, lesión de vía urinaria, lesión intestinal y tasa de conversión. Luego se 
analiza las complicaciones postoperatorias: médicas (presencia de fiebre, tromboembolismo, infección urinaria, neumonía, hematomas pélvicos, shock, sepsis e íleo), dolor postoperatorio (medido a través de una escala métrica de rango 0-10 de acuerdo a la intensidad del dolor referido por la paciente), necesidad de transfusión sanguínea, infección de herida operatoria, ingreso a Unidad de Cuidados Intensivos, necesidad de uso de analgesia endovenosa mayor de 24 horas, uso de antibióticos endovenoso (como tratamiento), proporción de pacientes que requirió exámenes postoperatorios, necesidad de reintervención y requerimiento de interconsultas a otras especialidades.

Posteriormente se realizó un estudio comparativo de los costos de producción de las diferentes modalidades quirúrgicas con los costos reales. El costo de producción se compone del gasto teórico que el hospital realiza para llevar a cabo un procedimiento, en este caso la histerectomía. Incluye los días de hospitalización, los exámenes de laboratorio, los procedimientos, los medicamentos e insumos requeridos durante la hospitalización, la intervención quirúrgica propiamente tal $\mathrm{y}$, en general, todos los procedimientos relacionados que significan algún costo para el hospital. Los costos reales también consideran todos los factores que se mencionan, pero se refieren a qué significa para el bolsillo del paciente la realización de la cirugía, que considera los gastos asociados a factores inherentes a las características de las pacientes sometidas a la cirugía y las demás variables señaladas previamente.

Las distintas variables fueron analizadas mediante el software estadístico STATA 8.0 a través de test de comparación de dos medias y test de Fisher para comparar proporciones. Las diferencias fueron consideradas estadísticamente significativas con $\mathrm{p}<0,05$.

\section{RESULTADOS}

El análisis final se realizó sobre un total de 1.663 pacientes sometidos a histerectomía en el período 1997-2005. Durante este tiempo se realizaron $1.474(88,63 \%)$ histerectomías abdominales (HA) y 189 (11,36\%) histerectomías laparoscópicas (HL). Los diagnósticos asociados a la intervención quirúrgica se muestran en la tabla I. La tabla II muestra el análisis de las distintas variables estudiadas.

La edad media de las pacientes sometidas a HA fue de 47,3 años y 46,3 años para la HL no presentando diferencias significativas. En relación al tiempo operatorio la HL requirió como tiempo medio 124 minutos y la HA 119 minutos no encontrándose diferencias estadísticamente significativas $(\mathrm{P}=0.9784)$.

Tampoco se encontraron diferencias significativas en relación a las complicaciones inmediatas derivadas de la cirugía (pérdida sanguínea importante, lesión vesical, lesión vía urinaria, lesión intestinal) $(\mathrm{P}=0.714)$. Estas fueron de $5,97 \%$ para HA y de $3,17 \%$ para HL. El porcentaje de conversión para la HL fue de $6,87 \%$.

Las complicaciones post operatorias, en su totalidad, se presentan de manera significativamente mayor en la HA $(\mathrm{P}<0.001)$.

Específicamente, el dolor postoperatorio moderado a severo fue significativamente mayor en la ruta abdominal $(\mathrm{P}<0.001)$. La necesidad de analgesia endovenosa por un tiempo mayor de 24 horas es concordante con lo anterior y fue significativamente mayor en la HA ( $\mathrm{P}<0.001)$.

Existen también diferencias significativas respecto del uso de tratamiento antibiótico endovenoso, siendo más requerido en las pacientes que se realizaron una HA. La infección de la zona operatoria (infección de sitio operatorio y accesos de trócares) fue mayor en la HA siendo esto estadísticamente significativo $(\mathrm{P}=0.042)$. Debido a su evolución, el 26,21\% de las 1.663 histerectomías requirió exámenes postoperatorios, siendo mayor en la HA, hecho estadísticamente significativo al compararlo con la HL $(\mathrm{P}<0.001)$. La realización de otros procedimientos quirúrgicos (tabla I) fue significativamente mayor en la HA $(\mathrm{P}<0.001)$.

La estadía hospitalaria promedio fue de 3,19 días para la HA y 2,39 días para la HL, diferencia estadísticamente significativa $(\mathrm{P}<0.0001)$.

$\mathrm{El}$ análisis de los costos muestra que el gasto en la HL es significativamente más elevado que 
Tabla 1. Principales diagnósticos preoperatorios en 1.663 pacientes sometidos a histerectomía por patología benigna según vía de acceso. Hospital Clínico Universidad de Chile, 1997-2005.

\begin{tabular}{|c|c|c|}
\hline $\begin{array}{l}\text { Diagnósticos } \\
\text { preoperatorios } n(\%)\end{array}$ & $\begin{array}{c}\text { Histerectomía } \\
\text { abdominal } \\
(n=1474)\end{array}$ & $\begin{array}{c}\text { Histerectomía } \\
\text { laparoscópica } \\
(n=189)\end{array}$ \\
\hline Mioma uterino & $993(67,36)$ & $153(80,95)$ \\
\hline Lesión anexial & $216(14,65)$ & $22(11,64)$ \\
\hline Adenomiosis & $93 \quad(6,30)$ & $17 \quad(8,99)$ \\
\hline Anemia secundaria & $53 \quad(3,59)$ & $5 \quad(2,64)$ \\
\hline Metrorragia/hipermenorrea & $\begin{array}{lll}\text { ea } & 47 & (3,18)\end{array}$ & $12 \quad(6,4)$ \\
\hline $\begin{array}{l}\text { Hiperplasia/engrosamiento } \\
\text { endometrial }\end{array}$ & to $40 \quad(2,71)$ & $18(9,52)$ \\
\hline Prolapso & $30(2,03)$ & $5(2,64)$ \\
\hline Endometriosis & $27(1,83)$ & $2(1,05)$ \\
\hline $\begin{array}{l}\text { Enfermedad inflamatoria } \\
\text { pélvica }\end{array}$ & $26(1,76)$ & $0 \quad(0,00)$ \\
\hline Absceso tuboovárico & $22(1,49)$ & $6 \quad(3,17)$ \\
\hline Algia pélvica & $18(1,22)$ & $0 \quad(0,00)$ \\
\hline
\end{tabular}

Algunos pacientes tienen múltiples diagnósticos y no necesariamente los diagnósticos son la indicación de la histerectomía. en la HA ( $\mathrm{P}<0.0001)$. El costo de producción de las histerectomías al compararlo con el costo real de cada una de las alternativas es significativamente menor $(\mathrm{P}<0.0001)$.

\section{DISCUSIÓN}

Está en discusión si la vía de abordaje para la histerectomía obedece a razones técnicas o es originada en preferencias del médico y/o equipo médico tratante ${ }^{28}$. La técnica laparoscópica tiene un costo mayor que la abdominal, si bien, éste puede reducirse al no utilizar material quirúrgico desechable ${ }^{26}$. No obstante, en los resultados de este estudio no se observan diferencias significativas en los costos ni en los tiempos operatorios, que según la literatura, explicarían estas diferencias ${ }^{26}$. Por esto, en nuestro hospital la vía de abordaje de la histerectomía dependería de otros factores como son la experiencia del equipo de cirujanos,

Tabla 2. Variables analizadas por vía de histerectomía. Hospital Clínico Universidad de Chile periodo 1997 - 2005.

\begin{tabular}{|c|c|c|c|}
\hline $\begin{array}{l}\text { Características de las } \\
\text { pacientes }(n=2527)\end{array}$ & $\begin{array}{c}\text { Histerectomía } \\
\text { abdominal(n=1474) }\end{array}$ & $\begin{array}{c}\text { Histerectomía } \\
\text { laparoscópica(n=189) }\end{array}$ & $\mathbf{p}$ \\
\hline $\begin{array}{l}\text { Promedio de edad } \pm \mathrm{DS} \\
\text { (rango en años) }\end{array}$ & $47,27 \pm 7,23(27-83)$ & $46,25 \pm 5,07(37-64)$ & 0.06 \\
\hline $\begin{array}{l}\text { Promedio tiempo operatorio } \\
\pm \text { DS (rango en minutos) }\end{array}$ & $118,98 \pm 34,28(30-240,5)$ & $124,48 \pm 42,11(55-360,1)$ & NS \\
\hline $\begin{array}{l}\text { Complicaciones inmediatas } \\
\%(n) *\end{array}$ & $5,97 \quad(88)$ & $3,17 \quad(6)$ & NS \\
\hline Conversión quirúrgica \% $(n)$ & no corresponde & $6,87(13)$ & No corresponde \\
\hline $\begin{array}{l}\text { Complicaciones post cirugía } \\
\%(n) \dagger\end{array}$ & $42,87(632)$ & $16,40(31)$ & $<0,001$ \\
\hline Dolor moderado a severo $\geq 4$ & $33,98(501)$ & $13,22(25)$ & 0.003 \\
\hline Transfusión & $0,81 \quad(12)$ & $0,52 \quad(1)$ & 1.000 \\
\hline Infección herida & $2,03 \quad(30)$ & $0,00 \quad(0)$ & 0.0042 \\
\hline Complicación médica $\%(n) \ddagger$ & $5,02 \quad(74)$ & $2,11 \quad(4)$ & 0.0097 \\
\hline Reoperación \% (n) & $1,01 \quad(15)$ & $0,52 \quad(1)$ & 1.000 \\
\hline $\begin{array}{l}\text { Estadía en Unidad de } \\
\text { Cuidados Intensivos } \%(n)\end{array}$ & $1,22 \quad(18)$ & $0,52 \quad(1)$ & $\mathrm{NS}$ \\
\hline $\begin{array}{l}\text { Estadía hospitalaria días } \pm \\
\text { DS rango (días) }\end{array}$ & $3,19 \pm 2,08(3-21)$ & $2,39 \pm 1,07(2-15)$ & $<0.0001$ \\
\hline Exámenes postoperatorios \% (n) & $17,97(265)$ & $5,82(11)$ & $<0.001$ \\
\hline $\begin{array}{l}\text { Tratamiento antibiótico } \\
\text { endovenoso }\end{array}$ & $14,99(221)$ & $4,23 \quad(8)$ & $<0.001$ \\
\hline Analgesia endovenosa $>24$ horas & $13,97(206)$ & $2,11 \quad(4)$ & $<0.001$ \\
\hline $\begin{array}{l}\text { Interconsulta especialidad } \%(n) \\
\text { En el postoperatorio }\end{array}$ & $5,97 \quad(88)$ & $3,17 \quad(6)$ & 0.175 \\
\hline Otros procedimientos $\%(n) \S$ & $16,01(236)$ & $3,17 \quad(6)$ & $<0.001$ \\
\hline $\begin{array}{l}\text { Costos reales en pesos chilenos } \\
\pm \text { DS (rango) }\end{array}$ & $\begin{array}{l}1.061 .879 \pm 105.847 \\
(956.033-1.167 .726)\end{array}$ & $\begin{array}{r}1.146 .082 \pm 112.322 \\
(1.033 .760-1.258 .404)\end{array}$ & $<0.0001$ \\
\hline $\begin{array}{l}\text { Costos teóricos de producción } \\
\text { en pesos chilenos }\end{array}$ & 928.446 & 928.446 & $<0.0001$ \\
\hline
\end{tabular}


la patología asociada y el tamaño uterino. Además, es sabido que el tamaño del mioma, la presencia de cicatrices o cirugía abdominal previa y la patología asociada, pueden ser un factor de sesgo al comparar los grupos analizados, lo que limita los resultados del estudio.

La tasa de complicaciones de nuestra serie no difiere de otras series ${ }^{29}$, al igual que los tiempos operatorios y los resultados de las otras variables estudiadas.

Actualmente, no disponemos de una revisión del seguimiento de las pacientes histerectomizadas en nuestro hospital, por lo que no obtuvimos datos en relación al retorno normal a las actividades y a la calidad de vida posterior al alta de las pacientes. Sin embargo, existen estudios previos de costo-efectividad que no demuestran mayores diferencias en la calidad de vida desde las primeras semanas de alta hasta meses posteriores a la cirugía ${ }^{10}$.

Como conclusión, es necesario señalar que en nuestro centro la histerectomía vaginal es la primera elección para la resolución de la patología uterina benigna cuando no presenta contraindicaciones. En este último escenario, la vía abdominal y la vía laparoscópica están indicadas y su elección depende en gran parte de la experiencia del equipo quirúrgico, variables como el tamaño uterino (muy discutida en la literatura) y la patología asociada.

\section{REFERENCIAS}

1. VESSEY MP, VILLARD-MACKINTOSH L, MCPHERSON K, COULTER A, YEATES D. The epidemiology of Hysterectomy: findings in a large cohort study. Br J Obstet Gynaecol 1992; 99: 402-7.

2. LEPINE LA, HILLIS SD, MARCHBANKS PA, KOONIN LM, MORROW B, KIEKE BA, et al. Hysterectomy surveillance-United States 19801993.MMWR Surveill Summ 1997; 46: 1-15.

3. GRAVES EJ, KOZAK LJ. National Hospital Discharge Survey: Annual Summary, 1996.

4. STOVALL T. Hysterectomy. Novak gynecology $13^{\text {th }}$ Ed. 2002; 22: 761-768.

5. CLINCH J. Length of Hospital stay after vaginal hysterectomy. Br J Obstet Gynaecol 1994; 101(3) 253-4.
6. GARRY R, FOUNTAIN J, MASON S, NAPP V, BRIDGMAN S, HAWE J, et al. The evaluate study: two parallel randomized trials, one comparing laparoscopic with abdominal hysterectomy, the other comparing laparoscopic with vaginal hysterectomy. BMJ 2004; 328(7432):129-33.

7. GARRY R, FOUNTAIN J, BROWN J, MANCA A, MASON S, SCULPHER M, et al. Evaluat hysterectomy trial: a multicentre randomized trial comparing abdominal, vaginal and laparoscopic methods of hysterectomy. Health Technology Assesment 2004; Vol8: No.26.

8. LIU CY, REICH H. Complications of total laparoscopic hysterectomy in 518 cases.Gynaecon Endosc 1994; 3: 203-208.

9. LUMSDEN MA, TWADDLE S, HAWTHORN R, TRAYNOR I, GILMORE D, DAVIS J, et al. A randomized comparison and economic evaluation of laparoscopic-assisted hysterectomy and abdominal hysterectomy. Br J Obstet Gynaecol 2000; 107(11): 1386-91.

10. JOHNSON N, BARLOW D, LETHABY A, TAVENDER E, CURR L, GARRY R. Methods of hysterectomy: systematic review and meta-analysis of randomized controlled trial. BMJ 2005; 330: 14781786.

11. WOOD C, MAHER P, HILL D, LOLATGIS N. Laparovaginal hysterectomy. Aust N Z J Obstet Gynaecol 1994; 34: 81-84.

12. Minelli L, ANGiOlillo M, CAiOnE C, PALMARA V. Laparoscopically assisted vaginal hysterectomy. Endoscopy 1991; 23: 64-66.

13. MENCAGLIA LUCA, HERENDAEL BV, TANTINI C, STAMPISI A. Laparoscopic assisted vaginal hysterectomy: evaluation of benefits of laparoscopic hysterectomy. Gynaecol Endosc 1994; 3 209-211.

14. HUNTER RW, MCCARTNEY AJ. Can laparoscopic assisted hysterectomy safely replace abdominal hysterectomy? Br J Obstet Gynaecol 1993; 100: 932-934.

15. BOIKE GM, ELFSTRAND EP, DELPRIORE G, et al. Laparoscopically assisted vaginal hysterectomy in a university hospital: report of 82 cases and comparison with abdominal and vaginal hysterectomy. Am J Osbtet Gynecol. 1993; 168:19601701.

16. KOVAK SR. Vaginal Hysterectomy. Bailliére's Clin Obstet Gynaecol. 1997; 11: 95-110.

17. DICKER RC, GREENSPAN JR, STRAUSS LT, et al. Complications of abdominal and vaginal hysterectomy among women of reproductive age in the United States. The Collaborative Review of Sterilization. Am J Obstet Gynecol. 1982; 144: 841-846.

18. KOVAC SR. Guidelines to determine the route of hysterectomy. Obstet Gynecol. 1995; 85: 18-23. 
19. RICHARDSON RE, BOAURNAS N, MAGOS A. Is a laparoscopic hysterectomy a waste of time? Lancet 1995; 345: 36-41.
20. QUERLEU D, COSSON M, PARMENTIER D, DEBODINANCE P. The impact of laparoscopic surgery on vaginal hysterectomy. Gynecol Endosc. 1993; 2: 89-91. 